\title{
Article \\ Electronic Structure of Tyrosyl D Radical of Photosystem II, as Revealed by 2D-Hyperfine Sublevel Correlation Spectroscopy
}

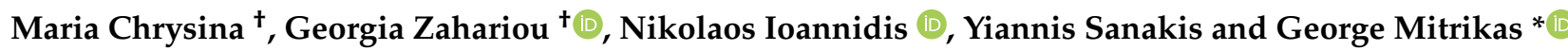 \\ Institute of Nanoscience and Nanotechnology, NCSR Demokritos, 15341 Athens, Greece; \\ m.chrysina@inn.demokritos.gr (M.C.); g.zahariou@inn.demokritos.gr (G.Z.); \\ n.ioannidis@inn.demokritos.gr (N.I.); i.sanakis@inn.demokritos.gr (Y.S.) \\ * Correspondence: g.mitrikas@inn.demokritos.gr \\ t These authors contributed equally to this work.
}

\section{check for} updates

Citation: Chrysina, M.; Zahariou, G.; Ioannidis, N.; Sanakis, Y.; Mitrikas, G. Electronic Structure of Tyrosyl D

Radical of Photosystem II, as

Revealed by 2D-Hyperfine Sublevel

Correlation Spectroscopy.

Magnetochemistry 2021, 7, 131.

https://doi.org/10.3390/

magnetochemistry7090131

Academic Editors: Angeliki

Giannoulis and Dinar Abdullin

Received: 7 August 2021

Accepted: 13 September 2021

Published: 21 September 2021

Publisher's Note: MDPI stays neutral with regard to jurisdictional claims in published maps and institutional affiliations.

Copyright: (c) 2021 by the authors. Licensee MDPI, Basel, Switzerland. This article is an open access article distributed under the terms and conditions of the Creative Commons Attribution (CC BY) license (https:// creativecommons.org/licenses/by/ $4.0 /)$.

\begin{abstract}
The biological water oxidation takes place in Photosystem II (PSII), a multi-subunit protein located in thylakoid membranes of higher plant chloroplasts and cyanobacteria. The catalytic site of PSII is a $\mathrm{Mn}_{4}$ Ca cluster and is known as the oxygen evolving complex (OEC) of PSII. Two tyrosine residues D1-Tyr161 $\left(\mathrm{Y}_{\mathrm{Z}}\right)$ and D2-Tyr160 $\left(\mathrm{Y}_{\mathrm{D}}\right)$ are symmetrically placed in the two core subunits D1 and D2 and participate in proton coupled electron transfer reactions. $\mathrm{Y}_{Z}$ of PSII is near the OEC and mediates electron coupled proton transfer from $\mathrm{Mn}_{4} \mathrm{Ca}$ to the photooxidizable chlorophyll species $\mathrm{P}_{680}^{+} . \mathrm{Y}_{\mathrm{D}}$ does not directly interact with OEC, but is crucial for modulating the various $\mathrm{S}$ oxidation states of the OEC. In PSII from higher plants the environment of $Y_{D} \bullet$ radical has been extensively characterized only in spinach (Spinacia oleracea) Mn-depleted non functional PSII membranes. Here, we present a 2D-HYSCORE investigation in functional PSII of spinach to determine the electronic structure of $\mathrm{Y}_{\mathrm{D}} \bullet$ radical. The hyperfine couplings of the protons that interact with the $\mathrm{Y}_{\mathrm{D}}{ }^{\bullet}$ radical are determined and the relevant assignment is provided. A discussion on the similarities and differences between the present results and the results from studies performed in non functional PSII membranes from higher plants and PSII preparations from other organisms is given.
\end{abstract}

Keywords: ESEEM; hyperfine sublevel correlation spectroscopy; photosystem II; proton hyperfine couplings

\section{Introduction}

Photosystem II (PSII) is a multi-subunit membrane-protein located within the thylakoid membranes of higher-plant chloroplasts, algae, and cyanobacteria. It utilizes solar energy to split two water molecules into molecular oxygen and protons, and produces reducing equivalents that are subsequently employed in carbon fixation reactions [1] The core of PSII consists of a pseudo-symmetric heterodimer of two homologous proteins D1 and D2. The catalytic site of PSII is a cluster located at D1 polypeptide and composed of four manganese ions and a calcium ion $\left(\mathrm{Mn}_{4} \mathrm{Ca}\right)$, known as the oxygen evolving complex (OEC) of PSII $[2,3]$. The OEC undergoes periodically four one-electron oxidation steps, $\mathrm{S}_{0}$ to $S_{1}, \ldots, S_{3}$ to $\left(S_{4}\right) S_{0}$, driven by the photooxidizable chlorophyll species $P_{680}$ [4].

Several cofactors participate in the electron coupled proton transfer reactions and contribute to the efficient function of PSII. Among them are the two tyrosine residues D1-Tyr161 $\left(\mathrm{Y}_{\mathrm{Z}}\right)$ and D2-Tyr160 $\left(\mathrm{Y}_{\mathrm{D}}\right)$, which are symmetrically placed in the two core subunits D1 and D2 [5-7]. During their oxidation, they form neutral $\mathrm{Y}_{\mathrm{Z}} \bullet$ and $\mathrm{Y}_{\mathrm{D}} \bullet$ radicals, because the proton of their phenolic group is transferred to groups found close by $[5,6,8,9]$. In addition, both of them are hydrogen bonded with a histidine molecule, His190 for $Y_{Z}$ and His189 for $Y_{D}[2,10]$. Despite the aforementioned similarities between $Y_{Z}$ and $Y_{D}$, they present important differences. The environment of $Y_{Z}$ is more hydrophilic, compared to that of $Y_{D}$ [11]. $Y_{Z}$ residue is in close vicinity to the OEC and is involved in fast electron 
coupled proton transfer reactions during water oxidation [2,3]. Particularly, the redoxactive tyrosine $Y_{Z}$ donates an electron to $P_{680}^{+}$at the rates of $30 \mathrm{~ns}$ to $50 \mu \mathrm{s}$ [5,12], while its reduction by $\mathrm{Mn}_{4} \mathrm{Ca}$ takes place at $30 \mu \mathrm{s}$ to $2 \mathrm{~ms}$, depending on the various $\mathrm{S}$ oxidation states of the OEC [13-15]. Instead, $Y_{D}$ is placed relatively far from the OEC $(\sim 30 \AA)$, and has only a weak interaction with it $[16,17]$. It also displays much slower kinetics, donating an electron to $\mathrm{P}_{680}^{+}$in the millisecond regime $[8,18]$. Additionally, the free radical $\mathrm{Y}_{\mathrm{D}}{ }^{\bullet}$ is importantly stable at room temperature and is reduced by the $\mathrm{S}_{0}$ state of the OEC in hours $[8,16,18]$.

Although $\mathrm{Y}_{\mathrm{D}}$ does not directly interact with $\mathrm{Mn}_{4} \mathrm{Ca}$ for the water splitting process, it plays important role for the efficient function of PSII [8,16,17,19-32]. Previous work on $\mathrm{Y}_{\mathrm{D}}$-less mutant of Synechocystis PSII showed that the redox properties of $\mathrm{Y}_{\mathrm{Z}}$ are altered [23]. In addition, the replacement of tyrosine $\mathrm{D}$ with Phenylalanine affects the proton transfer pathways for the reduction of $\mathrm{P}_{680}^{+}$in Chlamydomonas PSII cores [31]. These observations strongly indicate that tyrosine $\mathrm{D}$ has a significant influence on proton-coupled electron transfer events in the vicinity of $Y_{Z}$. While the electrostatic contribution of $Y_{D}$ to the reduction potential of redox couple $\mathrm{P}_{680}^{+} / \mathrm{P}_{680}$ is very small [30], tyrosine $\mathrm{D}$ affects the energetics of $\mathrm{P}_{680}^{+}$, accelerating the photoactivation process of the $\mathrm{Mn}_{4} \mathrm{CaO}_{5}$, and protecting against photoinhibition $[25,26,28,32]$. Additionally, $Y_{D}$ provides an oxidizing equivalent for the assembly of the $\mathrm{Mn}_{4} \mathrm{Ca}$ cluster [22,30], and serves functions related to modulating the various $\mathrm{S}$ oxidation states of the OEC, by maintaining it in stable higher valence states [25-27].

Owing to the functional role of $Y_{D}{ }^{\bullet}$ in Photosystem II, many efforts have been performed to characterize its structural environment and its electronic properties. As holds in all tyrosyl radicals, the unpaired electron is delocalized over the phenolic ring and the spin density distribution can be investigated via the hyperfine couplings of the 2,6- and 3,5 -protons, as well as the $\beta$-methylene protons, shown in Scheme 1 . In most cases, the continuous wave Electron Paramagnetic Resonance (cw-EPR) technique fails to describe in detail these couplings, owing to the spectral broadening from magnetic interactions of different origin. Instead, pulsed EPR spectroscopic techniques, including Electron-Spin Echo Envelope Modulation (ESEEM), Electron-Nuclear Double Resonance (ENDOR), 2 Dimensional Hyperfine Sublevel Correlation Spectroscopy (2D-HYSCORE) are useful tools for the study of these interactions and have been extensively used to characterize the hyperfine couplings of tyrosyl radicals [33-39]. In the case of cyanobacterial PSII, the most detailed description of the structural environment of $Y_{D}{ }^{\bullet}$ has been reached from an ENDOR study at W-band frequencies of single crystals PSII from Th. elongatus [35]. Regarding the higher plant PSII membranes, investigations by using the 2D-HYSCORE spectroscopy in spinach PSII preparations provided the most accurate determination of the hyperfine couplings of $Y_{D}{ }^{\bullet}$, but solely in unfunctional Mn-depleted membranes [33,36]. It should be noted that even if the cyanobacterial and the higher plant PSII present similar structural and functional similarities [40], some spectroscopic differences have been reported [41-44].

(a)

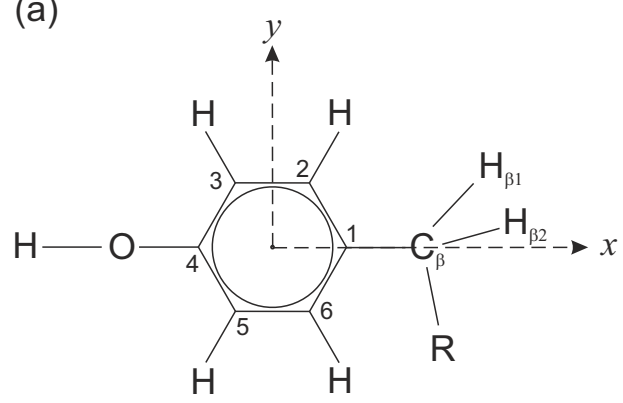

(b)

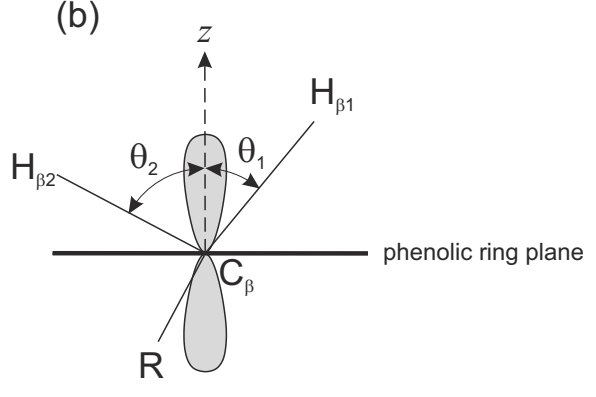

Scheme 1. Schematic representation of a tyrosine (a) and the dihedral angles $\theta_{1}, \theta_{2}$ of the $\beta$-methylene protons $(\mathbf{b})$. 
Here, we present a 2D-HYSCORE investigation in functional PSII of spinach poised in the $S_{1}$ state to determine the electronic structure of $Y_{D}{ }^{\bullet}$ radical. The experiments were performed in untreated and in $\mathrm{H}_{2} \mathrm{O} / \mathrm{D}_{2} \mathrm{O}$ exchanged PSII membranes. The spectra show characteristic peaks originating from the aromatic ring protons and $\beta$-methylene ones of $\mathrm{Y}_{\mathrm{D}}{ }^{\bullet}$. Our subsequent simulated analysis determine the hyperfine coupling constants of the proton nuclei with the free electron of $Y_{D} \cdot$ radical.

\section{Materials and Methods}

\subsection{Preparation of Spinach PSII Membranes}

PSII-enriched thylakoid membranes were prepared from spinach, following standard methods $[45,46]$. The samples were suspended in $400 \mathrm{mM}$ sucrose, $15 \mathrm{mM} \mathrm{NaCl}, 5 \mathrm{mM}$ $\mathrm{MgCl}_{2}, 40 \mathrm{mM}$ MES at $\mathrm{pH} 6.5$, at about 6-8 $\mathrm{mg} \mathrm{Chl} / \mathrm{mL}$. For the deuterium exchange of PSII the samples were pelleted by centrifugation and the pellet was diluted 10 times in deuterated buffer containing $400 \mathrm{mM}$ sucrose, $15 \mathrm{mM} \mathrm{NaCl}, 5 \mathrm{mM} \mathrm{MgCl}, 40 \mathrm{mM}$ MES in $\mathrm{D}_{2} \mathrm{O}$, at $\mathrm{pD}=6.5$. The membranes were centrifuged and resuspended in deuterated buffer twice. The total time that the sample was incubated in deuterated buffer at $4{ }^{\circ} \mathrm{C}$ was $5 \mathrm{~h}$.

\subsection{EPR Spectroscopy}

Cw EPR measurements at Q-band were carried out on a home-built spectrometer equipped with an ER 5106 QT Bruker resonator, an Anritsu MF76A microwave frequency counter and an Oxford Instruments helium cryostat. The temperature was controlled using an ITC 4 temperature controller. EPR measurements (cw and pulse modes) at X-band were performed on a Bruker ESP 380E spectrometer equipped with an EN 4118X-MD4 Bruker resonator and a HP 5350B microwave frequency counter. Measurements at cryogenic temperatures $(80 \mathrm{~K})$ were performed using a helium cryostat from Oxford Instruments. The temperature was stabilized using an Oxford Instruments ITC4 temperature controller. The field-swept EPR spectrum was recorded via free induction decay (FID) following a pulse length of $500 \mathrm{~ns}$. HYSCORE measurements employing the pulse sequence $\pi / 2-$ $\tau-\pi / 2-t_{1}-\pi-t_{2}-\pi / 2-\tau-$ echo were carried out with the following instrumental parameters: $t_{\pi / 2}=16 \mathrm{~ns} ; t_{\pi}=32 \mathrm{~ns}$; starting values of the two variable times $t_{1}$ and $t_{2}$, $56 \mathrm{~ns}$; time increment, $\Delta t=16 \mathrm{~ns}$ (data matrix $250 \times 250$ ). In order to eliminate blind-spot artifacts, up to five spectra were recorded with $\tau=96,112,136,160$, and $184 \mathrm{~ns}$. A fourstep phase cycle was used to remove undesired echoes. The data were processed with the program MATLAB (The MathWorks, Natick, MA, USA). The HYSCORE time traces were baseline corrected with a biexponential, apodized with a Gaussian window, and zero filled. After a two-dimensional Fourier transformation, the absolute-value spectra were calculated. The experimental cw EPR and HYSCORE spectra were simulated using the EasySpin package [47].

\subsection{Analysis of HYSCORE Spectra}

The analysis of HYSCORE spectra was done with the methodology developed by Dikanov et al. [48], which allows the accurate determination of isotropic and anisotropic hyperfine parameters without the need of complete spectrum simulations. This approach may also clarify whether different peaks belong to the same nuclei with nonaxial hyperfine tensors or not. For axial hyperfine interaction (HFI) with isotropic component $a$ and anisotropic tensor $(-T,-T, 2 T)$, i.e., $\left(A_{x}, A_{y}, A_{z}\right)=(a-T, a-T, a+2 T)$, the contour line shape in the powder 2D spectrum is described by [49]

$$
v_{\beta}=\left\{Q_{\beta} v_{\alpha}^{2}+G_{\beta}\right\}^{1 / 2},
$$

with

$$
Q_{\beta}=\frac{T+2 a+4 v_{I}}{T+2 a-4 v_{I}},
$$

and 


$$
G_{\beta}=-2 v_{I}\left(\frac{4 v_{I}^{2}-a^{2}+2 T^{2}-a T}{T+2 a-4 v_{I}}\right),
$$

where $v_{I}$ is the nuclear Zeeman frequency. The shape of this cross-peak in the $\left(v_{\alpha}, v_{\beta}\right)$ plot is an arc, but upon transforming to a $\left(v_{\alpha}^{2}, v_{\beta}^{2}\right)$ plot it is represented by a straight line with slope $Q_{\beta}$ and intercept $G_{\beta}$. From least square fitting of the $\left(v_{\alpha}^{2}, v_{\beta}^{2}\right)$ plot to a theoretical line the slope and the intercept can be calculated and thus, the values of $a$ and $T$ can be obtained. In the case of a rhombic hyperfine interaction, $\left(A_{x}, A_{y}, A_{z}\right)=(a-T-\delta, a-T+\delta, a+2 T)$, the HYSCORE plot forms three arcs. The same procedure can be followed for each one of the three arcs in order to obtain two of the three principal hyperfine coupling constants.

\section{Results and Discussion}

The experimental cw EPR spectra of $Y_{D}{ }^{\bullet}$ measured at $X$ - and Q-band frequencies are presented in Figure 1a,b, respectively. The cw spectra have approximate width of $\sim 16 \mathrm{G}$ from peak to valley and present a hyperfine structure. In order to reproduce the spectra the following spin Hamiltonian is considered

$$
\hat{\mathcal{H}}=\beta_{e} \mathbf{B}^{\mathrm{T}} \cdot \mathbf{g} \cdot \hat{\mathbf{S}}+\sum_{k=1}^{3}\left[\hat{\mathbf{S}}^{\mathrm{T}} \cdot \mathbf{A}_{\mathbf{k}} \cdot \hat{\mathbf{I}}_{\mathbf{k}}-g_{n} \beta_{n} \mathbf{B}^{\mathrm{T}} \cdot \hat{\mathbf{I}}_{\mathbf{k}}\right]
$$

where $\mathbf{g}$ and $g_{n}$ are the electron $g$-tensor and the proton $g$-factor, $\beta_{e}$ and $\beta_{n}$ are the Bohr and nuclear magnetons, $\mathbf{A}_{\mathbf{k}}$ is the hyperfine coupling tensor of the $k$-th proton, and $\mathbf{B}$ is the static magnetic field along $z$-axis. Three protons, namely 3,5 and $\beta_{1}$ were taken into account to reproduce the $\mathrm{cw}$ spectra. This involves a relatively large number of hyperfine parameters that cannot be unambiguously determined by the cw-spectra alone. The simulations (red lines) shown in Figurela,b were obtained using the hyperfine parameters determined by 2D-HYSCORE as it is discussed in detail below.
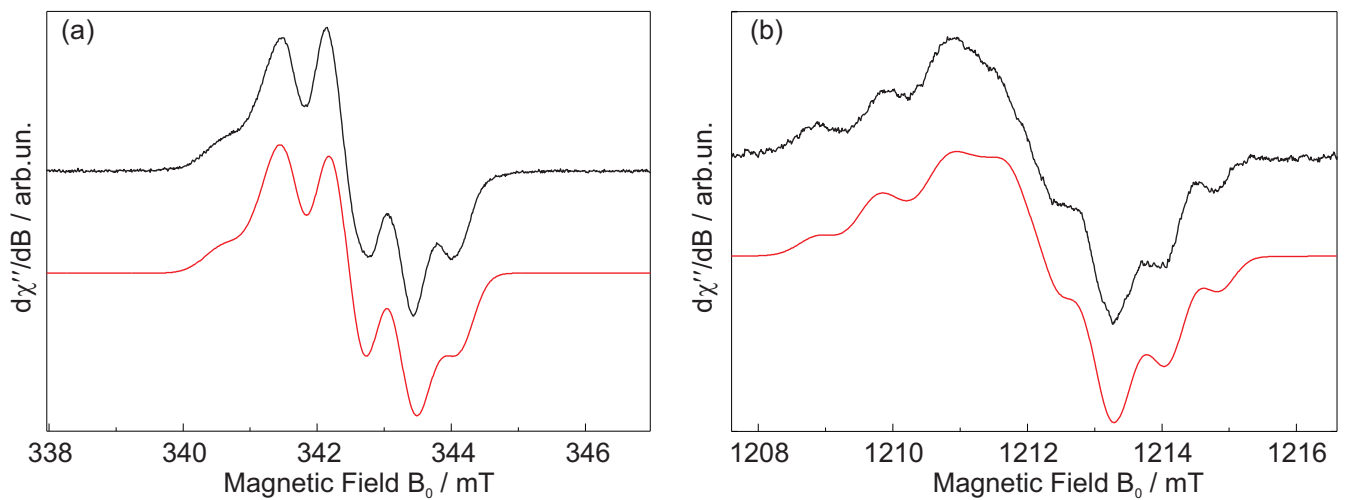

Figure 1. (a) $\mathrm{X}$-band and (b) Q-band $\mathrm{cw}$ EPR spectrum of $\mathrm{Y}_{\mathrm{D}}{ }^{\bullet}$. EPR parameters (a) microwave frequency, $9.60993 \mathrm{GHz}$; microwave power, $2 \mu \mathrm{W}$; modulation frequency, $100 \mathrm{kHz}$; modulation amplitude, $0.1 \mathrm{mT}$; temperature, $T=80 \mathrm{~K}$. (b) microwave frequency, $34.006 \mathrm{GHz}$; microwave power, $32.6 \mu \mathrm{W}$; modulation frequency, $100 \mathrm{kHz}$; modulation amplitude, $0.1 \mathrm{mT}$; temperature, $T=160 \mathrm{~K}$. Black traces: experiment. Red traces: simulations. Both spectra were simulated with $\left(g_{x}, g_{y}, g_{z}\right)=$ $(2.0075,2.0042,2.0020)$. For simulation details see text.

In a HYSCORE experiment, the $\pi / 2-\tau-\pi / 2$ subsequence creates nuclear coherence which evolves in the $\alpha(\beta)$ electron spin manifold during the first evolution period $t_{1}$. The nonselective $\pi$ pulse acts as a mixer that interchanges the nuclear coherence between the electron spin $\alpha$ and $\beta$ manifolds. During the second evolution period $t_{2}$, the transferred nuclear coherence evolves in the $\beta(\alpha)$ electron spin manifold and a nuclear coherence transfer echo is created at about $t_{1}=t_{2}$. This echo is modulated with the frequencies $\left(v_{\alpha}, v_{\beta}\right)$ of the nuclear transitions of the two $m_{s}$ manifolds. The 2D plot of the time domain 
$\left[t_{1}, t_{2}\right]$ is converted into a 2D frequency domain $\left[v_{1}, v_{2}\right]$ plot through the Fourier transform which results in correlation peaks at $\left(v_{\alpha}, v_{\beta}\right)$ and $\left(v_{\beta}, v_{\alpha}\right)$ [50]. Due to inherent suppression effects [51], systems with large hyperfine anisotropy require measurements with different $\tau$ values. In this work, we sum up HYSCORE spectra measured with five different $\tau$ values to minimize blind spots and acquire undistorted spectra.

In Figure 2 we present the HYSCORE spectrum of $Y_{D}{ }^{\bullet}$ at $80 \mathrm{~K}$. This spectrum was measured at $343.2 \mathrm{mT}$, where the intensity of the $\mathrm{Y}_{\mathrm{D}}{ }^{\bullet}$ signal is maximum. The presented HYSCORE spectrum is the sum of five spectra measured with five different $\tau$ values in order to eliminate blind spots. Peaks from three different types of magnetic nuclei are observed and their site on the map is marked as antidiagonals defined by $v_{2}=2 v_{I}-v_{1}$ : ${ }^{14} \mathrm{~N},{ }^{13} \mathrm{C}$ and ${ }^{1} \mathrm{H}$ with $v_{I}=1.06,3.68$, and $14.61 \mathrm{MHz}$, respectively. The signal from ${ }^{13} \mathrm{C}$ is due to the natural abundance of $1 \%$ of this isotope and the peak is due to weakly coupled $\mathrm{C}$ atoms of the matrix. The signal of the $\mathrm{N}$ originates from the amino-group of the tyrosyl radical and the $\mathrm{N}$ of the $\mathrm{H}$-bond partner to TyrD, His190. The full intensity of the matrix peaks of ${ }^{1} \mathrm{H}$ and ${ }^{14} \mathrm{~N}$ is outside of the scale of the contour plot as can be seen from the skyline projection of the 2D plot in Figure 2.

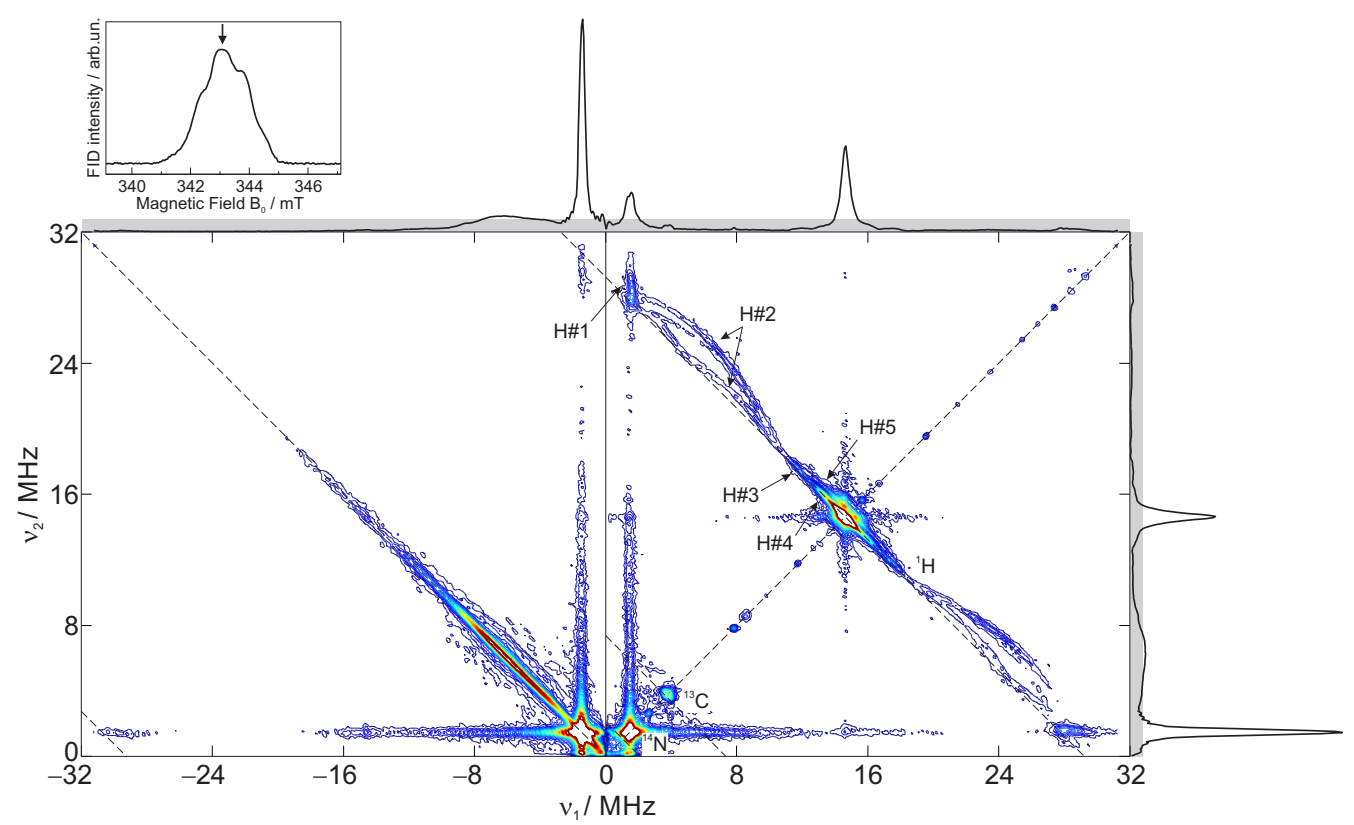

Figure 2. Contour plot of the experimental HYSCORE spectrum of $\mathrm{Y}_{\mathrm{D}}{ }^{\bullet}$ in PSII. The plot is the sum of five spectra measured with $\tau=96,112,136,160$, and 184 ns. Experimental parameters: magnetic field, $343.2 \mathrm{mT}$; microwave frequency, $9.629 \mathrm{GHz}$; temperature, $80 \mathrm{~K}$; time interval between successive pulse sets, $1 \mathrm{~ms}$. Gray areas in skyline projection denote the range of contour levels for the 2D plot. Inset: FID-detected EPR spectrum with observer position marked by the arrow.

We will focus on the ${ }^{1} \mathrm{H}$ nuclei that are the most well resolved and thus informative. By inspection of the spectrum five types of protons interacting with the electronic spin are observed, they are mainly on the $[+,+]$ quadrant and thus represent weak interaction with the electronic spin $\left(A<2 v_{I}\right.$, where $A$ : hyperfine coupling constant). The stronger the coupling is, the larger is the distance of the peaks from the diagonal. The peaks that correspond to stronger coupled protons are presented in more detail in Figure 3 (peaks $\mathrm{H \# 1}$ and $\mathrm{H \# 2}$ ) while the weaker coupled proton peaks are presented in Figure 4 (peaks $\mathrm{H \# 3-H \# 5).}$

The correlation peaks $\mathrm{H \# 1}$ around $[2,28] \mathrm{MHz}$ and $[28,2] \mathrm{MHz}$ stem from a proton with axial hyperfine coupling tensor and correspond to the strongest observed interaction. This proton gives weaker intensity peaks also at the $[-,+]$ quadrant because its coupling is close to the exact cancelation condition $\left(A=2 v_{I}\right)$. The three arc-shaped correlation ridges $\mathrm{H \# 2}$, that extend between [2-10,28-19] MHz and [19-28,10-2] MHz, originate from proton 
hyperfine couplings with rhombic symmetry. They cross to each other on the antidiagonal and from these cross points the principal hyperfine coupling constants $A_{x}, A_{y}$, and $A_{z}$ can be inferred. In Figure 3a the stronger couplings (peaks $\mathrm{H \# 1}$ and $\mathrm{H \# 2}$ ) are presented in detail. Ridges from two rhombic protons are observed as can be seen from the two long arcs ranging between [2-10,19-28] MHz and [1-10,19-28] MHz, which correspond to two different sets of principal values (say $A_{x}, A_{z}$ ). The small arc in the frequency region [3-6,23-25] MHz, that define the third hyperfine coupling component, $A_{y}$, does not cross the ${ }^{1} \mathrm{H}$ antidiagonal in a single point. This implies that it is not a single ridge, but two. These two protons have slightly different hyperfine coupling values that can not be distinguished in a cw-EPR or ENDOR powder spectrum. Here, we show that HYSCORE spectroscopy provides maximum resolution that allows for inspecting such small differences.

The three peaks (H\#3-H\#5) lying close to the diagonal originate from weakly coupled protons. They are not completely resolved because they partially overlap with the strong intensity peak of the matrix protons. Due to the lack of optimum resolution in this frequency range, these couplings are treated as axial in the following analysis, although a small rhombicity can not be excluded.
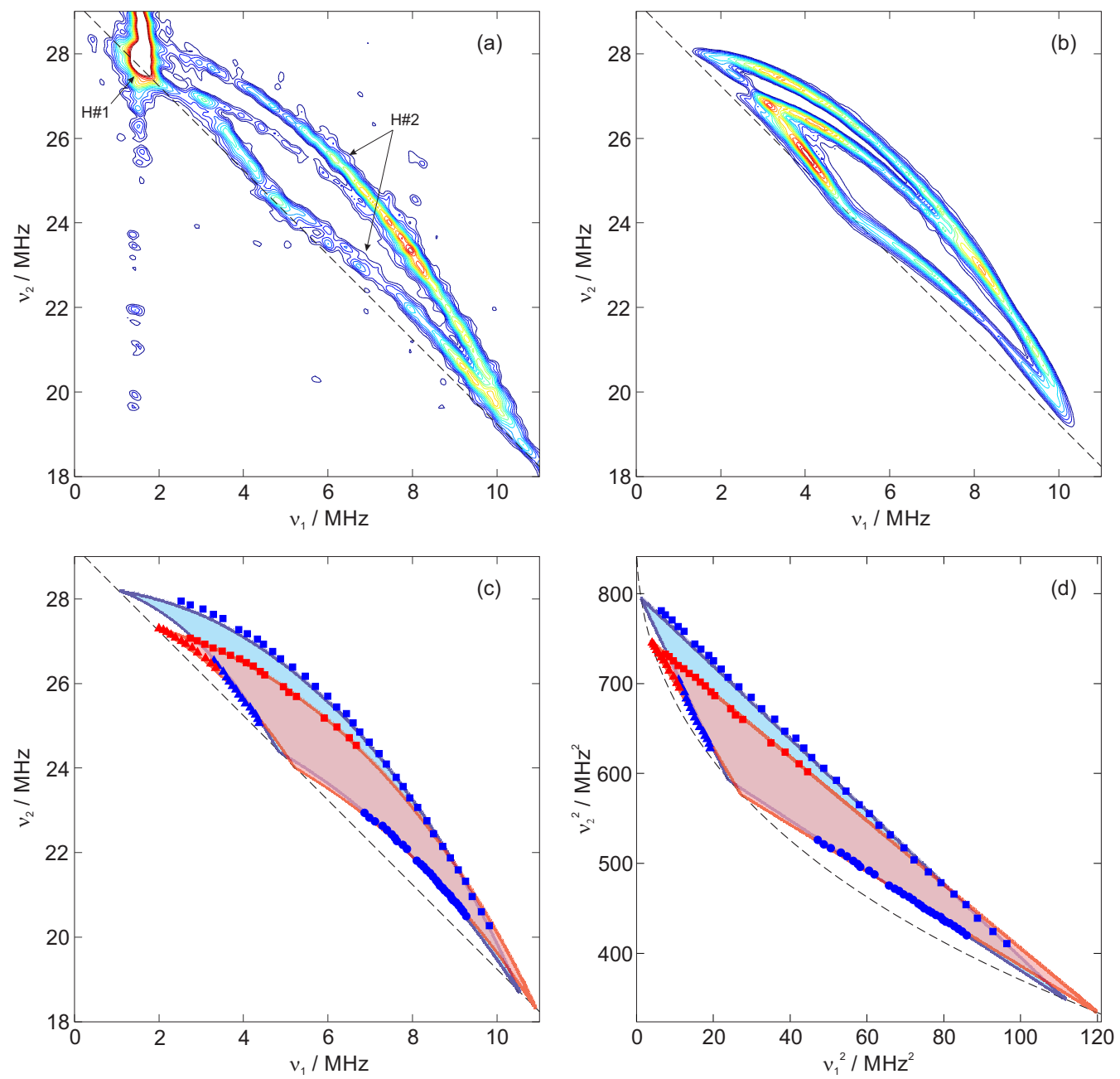

Figure 3. (a) Detail of the HYSCORE spectrum showing correlation ridges of $\beta$-type methylene (H\#1) and 3,5-protons (H\#2). (b) Full HYSCORE simulation of H\#2 using the parameters listed in Table 1. (c) Selected points from the experimental spectrum (filled symbols) and the corresponding fits (solid curves) using Dikanov analysis. Shaded areas in two different colors represent the two different sets of ${ }^{1} \mathrm{H}$ principal hyperfine coupling constants obtained from this analysis. (d) Data as in (c) but in the $\left(v_{1}^{2}, v_{2}^{2}\right)$ representation. 

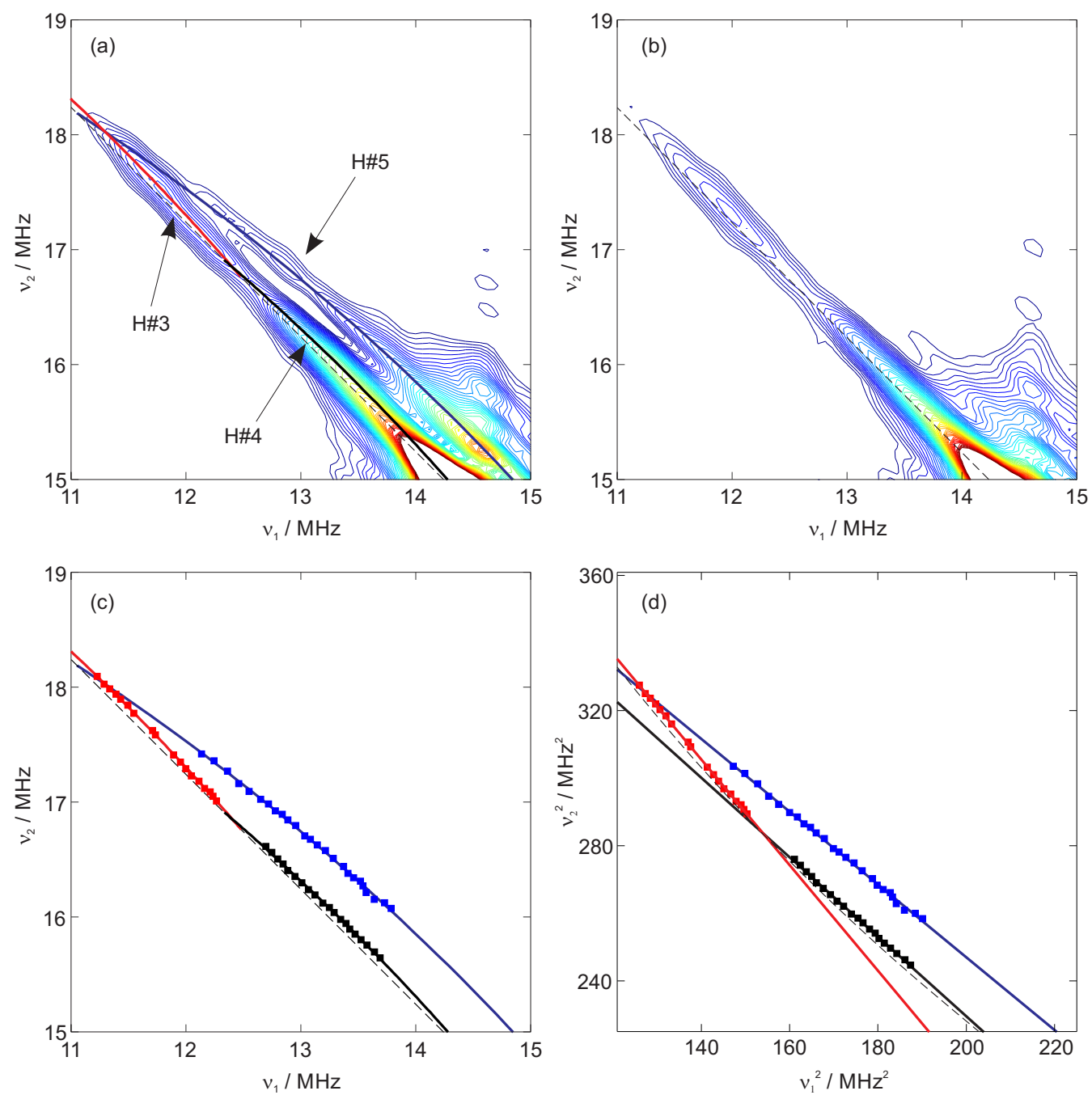

Figure 4. (a) Detail of the HYSCORE spectrum showing correlation ridges H\#3, H\#4, and H\#5 lying close to the diagonal. Solid curves represent fitted data using Dikanov analysis. (b) HYSCORE spectrum of the TyrD radical in deuterated buffer. (c) Selected points from the experimental spectrum (a) (filled squares) and the corresponding fits (solid curves) using Dikanov analysis. (d) Data as in (c) but in the $\left(v_{1}^{2}, v_{2}^{2}\right)$ representation.

Determination of the principal values of hyperfine coupling constant by Dikanov analysis. The principal values of the hyperfine coupling constant of the five types of nuclei were calculated by Dikanov analysis. As described in the Methods section, this analysis converts the arc-shaped ridges of an axial hyperfine coupling tensor of the $\left(v_{1}, v_{2}\right)$ plot to a linear function in the $\left(v_{1}^{2}, v_{2}^{2}\right)$ plot. From the slope and intercept of the fitted linear function the isotropic component, $a$, and anisotropic component, T, can be inferred. For H\#1, two possible solutions with $a=27.9 \mathrm{MHz}$ and $T=2 \mathrm{MHz}$ or $a=-29.9 \mathrm{MHz}$ and $T=2 \mathrm{MHz}$ are obtained. Thus, the principal values of the hyperfine coupling tensor are $\left|A_{\perp}\right|=25.9 \mathrm{MHz}$ and $\left|A_{\|}\right|=31.9 \mathrm{MHz}$ or $\left|A_{\perp}\right|=31.9 \mathrm{MHz}$ and $\left|A_{\|}\right|=25.9 \mathrm{MHz}$. Based on the assignment of this coupling to one of the two $\beta$-methylene protons (vide infra) which implies $a>0$, and the full simulation of this signal, the second option is ruled out.

For the case of nonaxial hyperfine interaction a similar analysis can be applied as follows: each one of the three arcs ridges that correspond to orientations of the magnetic field perpendicular to each of the principal axes of the hyperfine interaction tensor [48,49], can be treated as an axial system which allows two of the three principal values to be determined. The process can be repeated on the other two arcs and, provided that the estimated values can be matched in pairs, the three principal values of the tensor can be determined. In this case, the three fitted straight lines should form a triangle in the $\left(v_{1}^{2}, v_{2}^{2}\right)$ 
representation, with the apexes lying on the $\left|v_{1}+v_{2}\right|=2 v_{I}$ curve. Importantly, this is a criterion to discriminate couplings from different nuclei. For instance, the two long ridges marked by square symbols in Figure 3c must belong to protons with different hyperfine coupling tensors because their corresponding fitted straight lines in Figure $3 \mathrm{~d}$ cross each other away from the dashed curve. Moreover, the combination of data from the other two ridges (circle and triangle symbols), gives the two sets of principal values for H\#2 peaks listed in Table 1. Although there is a small uncertainty for one of the principal values (i.e., $\left|A_{y}\right|=8 \mathrm{MHz}$ ) of the red-coloured peaks due to the overlap with other peaks, the excellent agreement between the experimental spectrum of Figure 3 a and the full simulation shown in Figure $3 b$ provides good confidence for the extracted parameters.

In Figure $4 \mathrm{a}$ the peaks from weakly coupled protons are presented. Upon deuteration of the buffer the long ridge H\#5 disappears as can be seen in Figure $4 \mathrm{~b}$ which means that this signal stems from an exchangeable proton interacting with the electronic spin. Additionally, the ${ }^{2} \mathrm{H}$ matrix peak at $[2.2,2.2] \mathrm{MHz}$ is also observed in the HYSCORE spectrum (not shown). The selected data for the Dikanov analysis of H\#3, H\#4 and H\#5 peaks are shown in Figure $4 \mathrm{c}, \mathrm{d}$ and the obtained hyperfine coupling constants are listed in Table 1.

Table 1. Proton hyperfine couplings in the g-frame $(x, y, z)$ (as defined in Scheme 1) obtained from the analysis of HYSCORE spectra in this work.

\begin{tabular}{ccccccccc}
\hline \multicolumn{1}{c}{ Hyperfine Coupling Constants in $\mathbf{M H z}{ }^{*}$} \\
Peak No. & $\boldsymbol{A}_{\boldsymbol{x}}$ & $\boldsymbol{A}_{\boldsymbol{y}}$ & $\boldsymbol{A}_{\boldsymbol{z}}$ & $\boldsymbol{a}$ & $\boldsymbol{T}$ & $\boldsymbol{\delta}$ & $\boldsymbol{\rho}_{\boldsymbol{C}}$ & Assignment \\
\hline H\#1 & 32.0 & 25.9 & 25.9 & 27.9 & 2.0 & & 0.38 & $\beta_{1}$ \\
H\#2 & -27.5 & -8.1 & -19.9 & -18.5 & 5.2 & 3.8 & 0.27 & $3^{* *}$ \\
H\#2 & -25.5 & -8.0 & -19.1 & -17.5 & 4.8 & 3.1 & 0.25 & $5^{* *}$ \\
H\#3 & 8.6 & 4.3 & 4.3 & 5.7 & 1.4 & & -0.08 & $2,6, \beta_{2}$ \\
H\#4 & 4.6 & 0.2 & 0.2 & 1.7 & 1.5 & & & protein \\
H\#5 & 7.2 & -4.8 & -4.8 & -0.8 & 4.0 & & & H-bond \\
\hline
\end{tabular}

* Signs were adjusted according to previous results of single-crystal [35] and theoretical [11] studies. ${ }^{* *}$ For 3,5 protons an Euler angle $\alpha$ of $23^{\circ}$ and $-23^{\circ}$ between the $\mathbf{A}$ tensor and the $\mathrm{g}$-frame was assumed.

Assignment of the cross peaks of the HYSCORE spectrum to protons of the TyrD radical. Hyperfine couplings of the protons of TyrD radical represent how strongly the electronic spin interacts with these protons and thus reflect the distribution of the spin over the radical. For radicals with $\pi$ conjugated systems the spin density is delocalized over the $\pi$ orbitals and for tyrosine mainly over the phenolic ring. The electron spin density is not equally distributed over the whole ring; theoretical calculations reveal higher spin density on carbon atoms 3,5 than on 2,6 [52], thus stronger hyperfine couplings are expected for the corresponding 3,5 protons than the 2,6 protons. Moreover, due to the symmetry of the molecule, the hyperfine coupling tensors of the phenolic ring protons have considerable nonaxial character. Based on these arguments, $\mathrm{H \# 2}$ peaks are attributed to 3 and 5 protons of the phenolic ring for which strong anisotropic couplings are expected. The small difference in $a, T$ and $\delta$ between these two protons reflects the breaking of symmetry about $C_{1}-C_{4}$ axis due to the local environment of the radical, and demonstrates the power of HYSCORE spectroscopy. On the other hand, $\mathrm{H \# 3}$ peaks are ascribed to protons 2 and 6 because their obtained parameters are compatible with the reported smaller hyperfine couplings [33,35].

For the $\alpha$-protons of the phenolic ring the unpaired spin density occurs through spin polarization mechanisms due to the exchange interaction between the electrons in the $\mathrm{C}-\mathrm{H} \sigma$ bond and the $\pi$ electrons [53]. The isotropic coupling $a(\mathrm{H})$ is related to the spin population at the carbon $2 p_{z}$ orbital, $\rho_{p_{z}, C}$, through the McConnell equation

$$
a(H)=Q \rho_{p_{z}, C}
$$

with $Q=-69.9 \mathrm{MHz}$ [54]. The determined spin populations of carbon atoms 2, 3, 5, and 6 using the latter equation are listed in Table 1. 
For methylene protons ( $\beta$-protons), the mechanism responsible for the unpaired spin density is called hyperconjugation. For a positive spin population $\rho_{C_{1}}$ at carbon $C_{1}$, this mechanism gives positive hyperfine coupling [55]

$$
a\left(H-C_{\beta}\right)=B_{2} \rho_{C_{1}} \cos ^{2} \theta,
$$

where $B_{2}=162 \mathrm{MHz}$ is a semiempirical constant [56], and $\theta$ is the dihedral angle defined by the direction of the $p_{z}$ orbital on $C_{1}$, the $C_{1}-C_{\beta}$ bond and the direction of the proton bound to $C_{\beta}$ (Scheme 1). The phenolic ring can rotate around the $C_{1}-C_{\beta}$ axis and this rotation modulates the $a$ values of the two $\beta$-protons. The orientation of the ring depends on the proteinic environment of tyrosine residue. Therefore, hyperfine coupling values of $\beta$ protons are enzyme- and species-dependent. Previous studies $[35,36,57]$ showed that one of the two methylene protons $\left(\beta_{2}\right)$ is closer to the phenolic ring plane and thus has considerably smaller $a_{2}$ compared to that of $\beta_{1}$. The large isotropic coupling constant $a_{1}=27.9 \mathrm{MHz}$ obtained for the $\mathrm{H \# 1}$ peak is in line with the typical values $23-30 \mathrm{MHz}$ assigned to $\beta_{1}$ proton. On the other hand, the HYSCORE peaks of the $\beta_{2}$-proton should appear within the limits of the spectrum shown in Figure 4a. Although the H\#4 peak corresponds to a weak hyperfine coupling, it is unlikely to belong to $\beta_{2}$ proton which should be dominated by the isotropic term with the dipolar term being small (10-20\% of isotropic) by comparison. Based on this argument and the relatively strong intensity of this signal, the H\#4 peak is tentatively assigned to weakly-coupled protons of the proteinic environment.

The lack of additional peaks in Figure 4a that could be assigned to the $\beta_{2}$ proton implies its overlap with the peak $\mathrm{H \# 2}$ (ring protons 2,6 ) and a similarity between their magnetic parameters. From the center of peak $\mathrm{H \#} 2$ we estimate $a_{2}=5.4 \mathrm{MHz}$ that can be combined with $a_{1}=27.9 \mathrm{MHz}$ in order to estimate $\rho_{\mathrm{C}_{1}}$ as follows: inserting the ratio $a_{1} / a_{2}=5.17$ into Equation (6) and assuming $\left|\theta_{1}-\theta_{2}\right|=120^{\circ}$, we calculate $\theta_{1}=47^{\circ}$, $\theta_{2}=-73^{\circ}$ and $\rho_{C_{1}} B=60.9 \mathrm{MHz}$, from which we find $\rho_{C_{1}}=0.38$.

Peak $\mathrm{H \# 5}$ is attributed to the phenolic proton of the ring that is exchangeable upon deuteration of the buffer. The obtained dipolar hyperfine coupling $T=3.98 \pm 0.20 \mathrm{MHz}$ can be utilized to determine the radial distance $r$ between the electron spin and the exchangeable proton through the point-dipole approximation

$$
T=\rho_{\mathrm{O}} \frac{\mu_{0}}{4 \pi h} \frac{g \beta_{e} g_{n} \beta_{n}}{r^{3}}
$$

where $g$ and $g_{n}$ are the electron and ${ }^{1} \mathrm{H}$ nuclear $\mathrm{g}$ factors, respectively, $\beta_{e}$ and $\beta_{n}$ are the Bohr and nuclear magnetons,respectively, and $\rho_{\mathrm{O}}$ is the unpaired spin density on the tyrosyl oxygen. Using the values of spin densities listed in Table1, we estimate $\rho_{\mathrm{C}_{4}+\mathrm{O}}=0.26$, and assuming $\rho_{\mathrm{C}_{4}}=0$, we finally obtain $r=1.73 \pm 0.03 \AA$. Similar distance was calculated for Mn-depleted PS II from spinach using ENDOR (1.67 $\AA$, [58]), while longer distances for the PS II from T. elongatus (1.84 $\AA$, [59]) and Synechocystis (1.87-1.9 $\AA$, [36,58]). No indication for a second H-bond was found, which is in line with earlier reports $[36,58,59]$.

Comparison with earlier studies on TyrD radical. Various investigations that describe the hyperfine couplings of the protons interacting with the TyrD radical in intact PS II of spinach [60,61] and T. elongatus [35,62] have been reported. Except in [60], in which ENDOR at $14 \mathrm{GHz}$ was performed, these studies were conducted using high field EPR (90-140 GHz) [61,62] or high field EPR and ENDOR [35]. In high field, both EPR and ENDOR spectra have higher resolution and the couplings can be resolved with high accuracy. Interestingly, the two studies in T. elongatus are in single crystals $[35,62]$. This experiment would not be feasible in plant PS II because plant PS II is degraded during crystallization [63]. However, HYSCORE as a 2D technique can resolve the strong couplings (3,5 and the strong $\beta$ proton) with similar accuracy as the W-band ENDOR technique performed in single-crystal ([35], Table 2) and thus do without the need for crystals and long measurements in multiple angles. This fact proves HYSCORE as a powerful 
alternative for the study of the environment of radicals and in some metal ions as well, in powder samples. Of course, there are limitations on the information that can be extracted from HYSCORE (orientation and sign of the couplings are not defined, weak couplings are partially obscured by the matrix proton peak) and combination with other experiments is required.

TyrD radical has been studied by using HYSCORE in a variety of organisms: in spinach [33], in the green alga Chlamydomonas reinhardtii [57] and in the cyanobacterium Synechocystis [36], but only in Mn depleted PS II. It should be noted that in the previous studies one $\tau$ value was used and there are blind spots at the position of the $A_{y}$ coupling of the rhombic proton limiting the ability to determine this component accurately. The coupling of the $\beta_{1}$ proton is in good agreement with the $\beta_{1}$ proton of the Mn-depleted PS II from spinach [33] which suggests that the presence of Mn-cluster does not affect the position of the phenolic ring with respect to the $C_{1}-C_{\beta}$ axis. By inspection of the spectra in the above-mentioned studies and herein it is obvious that while the rhombic protons are similar (although not defined as two in [33,57]), the orientation of the ridge of the $\beta_{1}$ proton in relation to the antidiagonal is different in Synechocystis [36] than in higher plants ([33] and this study) and in green algae [57]. This difference is obtained by the direct comparison of the peak $\mathrm{H}^{I}$ shown in Figure 2 of ref. [36] with the peak H\#1 in Figure 2 of the present work, and is also reflected in the different $a$ values of $25.9 \mathrm{MHz}$ and $27.9 \mathrm{MHz}$, respectively.

The hyperfine coupling constants of TyrD radical determined in the present investigation are in good agreement with the values calculated for the "3ox" model in a study presented by Pantazis and co-workers [11] (Table 2). In that model, the water molecule adjacent to TyrD occupies the distal position (the distance of the water molecule to the phenolic $\mathrm{O}$ is $4.2 \AA$ ) and thus TyrD is H-bonded only to His 189 . Crystal structure studies $[2,3]$ revealed two possible positions for this water molecule, the "proximal" to TyrD and the "distal". In the former case, TyrD is hydrogen bonded to both His189 and the water molecule, while in the latter one only to His189. In that study it was found that the calculated hyperfine couplings of the strongly coupled $\beta$ proton decrease upon increasing the distance of the water from the phenolic $\mathrm{O}$, reflecting the local environment.

Table 2. Overview of reported proton hyperfine couplings (in $\mathrm{MHz}$ ) for the TyrD radical.

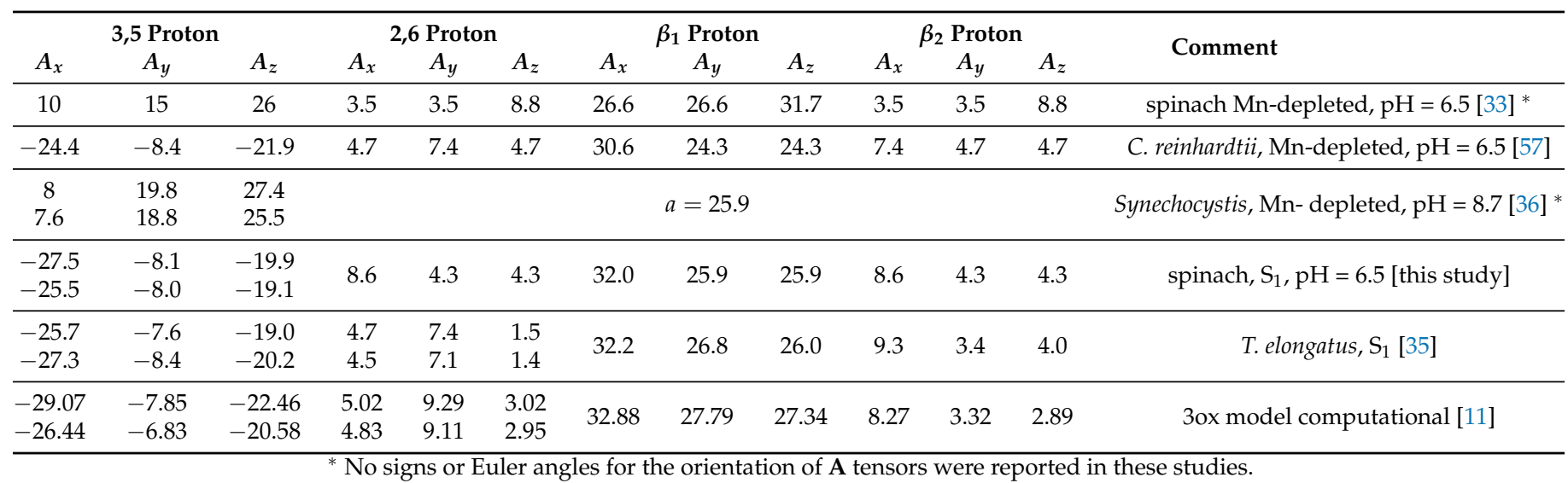

\section{Conclusions}

In this investigation, we present a 2D-HYSCORE study of $Y_{D} \bullet$ in functional spinach PSII, in order to determine its electronic structure. The experiments were carried out in untreated and in $\mathrm{H}_{2} \mathrm{O} / \mathrm{D}_{2} \mathrm{O}$ exchanged PSII preparations. The subsequent simulated analysis of the spectra accurately determine the hyperfine couplings of the free electron of $Y_{D}{ }^{\bullet}$ with the proton nuclei. The comparison of our results with those in Mn-depleted PSII preparations reveals significant similarities. This means that the presence of the Mncluster does not affect the electronic structure of $Y_{D}{ }^{\bullet}$. Instead, the signal assigned to the 
strongly coupled $\beta$-proton of $\mathrm{Y}_{\mathrm{D}}{ }^{\bullet}$ in spinach PSII is different from the respective signal from Synechocystis, implying structural differences between the various PSII organisms.

Author Contributions: Conceptualization, M.C., G.Z., N.I., Y.S. and G.M.; methodology, M.C., G.Z., N.I., Y.S. and G.M.; formal analysis, M.C., G.Z. and G.M.; investigation, M.C., G.Z., N.I. and G.M.; resources, Y.S. and G.M.; writing-review and editing, G.Z., M.C., N.I., Y.S. and G.M.; supervision, Y.S. and G.M.; project administration, G.M.; funding acquisition, Y.S. and G.M. All authors have read and agreed to the published version of the manuscript.

Funding: This research is co-financed by Greece and the European Union (European Social FundESF) through the Operational Programme "Human Resources Development, Education and Lifelong Learning 2014-2020" in the context of the project "Photosynthetic Water Splitting: The Critical Stages before Oxygen Release" (MIS 5047814).

Acknowledgments: We acknowledge support from the facility "Helium Liquefier and Low Temperature Spectroscopy", ELKE \#11 143, NCSR Demokritos.

Conflicts of Interest: The authors declare no conflict of interest.

\section{References}

1. Cox, N.; Pantazis, D.A.; Lubitz, W. Current Understanding of the Mechanism of Water Oxidation in Photosystem II and Its Relation to XFEL Data. Annu. Rev. Biochem. 2020, 89, 795-820. [CrossRef] [PubMed]

2. Umena, Y.; Kawakami, K.; Shen, J.; Kamiya, N. Crystal structure of oxygen-evolving photosystem II at a resolution of $1.9 \AA$. Nature 2011, 473, 55-60. [CrossRef]

3. Suga, M.; Akita, F.; Hirata, K.; Ueno, G.; Murakami, H.; Nakajima, Y.; Shimizu, T.; Yamashita, K.; Yamamoto, M.; Ago, H.; et al. Native structure of photosystem II at 1.95 angstrom resolution viewed by femtosecond X-ray pulses. Nature 2015, 517, 99-103. [CrossRef]

4. Kok, B.; Forbush, B.; McGloin, M. Cooperation of Charges in Photosynthetic $\mathrm{O}_{2}$ Evolution-I. A Linear Four Step Mechanism. Photochem. Photobiol. 1970, 11, 457-475. [CrossRef]

5. Diner, B.; Britt, R. The redox-active tyrosine $\mathrm{Y}_{\mathrm{z}}$ and $\mathrm{Y}_{\mathrm{D}}$. In Photosystem II: The Light-Driven Water: Plastoquinone Oxidoreductase; Wydrzynski, T., Satoh, K., Eds.; Springer: Dordrecht, The Netherlands, 2005; pp. 207-233.

6. Styring, S.; Sjöholm, J.; Mamedov, F. Two tyrosines that changed the world: Interfacing the oxidizing power of photochemistry to water splitting in photosystem II. Biochim. Biophys. Acta Bioenerg. 2012, 1817, 76-87. [CrossRef] [PubMed]

7. Koulougliotis, D.; Tang, X.; Diner, B.; Brudvig, G. Spectroscopic Evidence for the Symmetric Location of Tyrosines D and Z in Photosystem II. Biochemistry 1995, 34, 2850-2856. [CrossRef] [PubMed]

8. Faller, P.; Debus, R.J.; Brettel, K.; Sugiura, M.; Rutherford, A.W.; Boussac, A. Rapid formation of the stable tyrosyl radical in photosystem II. Proc. Natl. Acad. Sci. USA 2001, 98, 14368-14373. [CrossRef] [PubMed]

9. Nakamura, S.; Noguchi, T. Infrared Detection of a Proton Released from Tyrosine YD to the Bulk upon Its Photo-oxidation in Photosystem II. Biochemistry 2015, 54, 5045-5053. [CrossRef]

10. Saito, K.; Shen, J.R.; Ishida, T.; Ishikita, H. Short Hydrogen Bond between Redox-Active Tyrosine YZ and D1-His190 in the Photosystem II Crystal Structure. Biochemistry 2011, 50, 9836-9844. [CrossRef] [PubMed]

11. Sirohiwal, A.; Neese, F.; Pantazis, D.A. Microsolvation of the Redox-Active Tyrosine-D in Photosystem II: Correlation of Energetics with EPR Spectroscopy and Oxidation-Induced Proton Transfer. J. Am. Chem. Soc. 2019, 141, 3217-3231. [CrossRef]

12. Renger, G.; Renger, T. Photosystem II: The machinery of photosynthetic water splitting. Photosynth. Res. 2008, 98, 53-80. [CrossRef] [PubMed]

13. Rappaport, F.; Blanchard-Desce, M.; Lavergne, J. Kinetics of electron transfer and electrochromic change during the redox transitions of the photosynthetic oxygen-evolving complex. Biochim. Biophys. Acta Bioenerg. 1994, 1184, 178-192. [CrossRef]

14. Haumann, M.; Liebisch, P.; Müller, C.; Barra, M.; Grabolle, M.; Dau, H. Photosynthetic O2 Formation Tracked by Time-Resolved X-ray Experiments. Science 2005, 310, 1019-1021. [CrossRef] [PubMed]

15. Klauss, A.; Haumann, M.; Dau, H. Seven Steps of Alternating Electron and Proton Transfer in Photosystem II Water Oxidation Traced by Time-Resolved Photothermal Beam Deflection at Improved Sensitivity. J. Phys. Chem. B 2015, 119, 2677-2689. [CrossRef]

16. Styring, S.; Rutherford, A.W. In the oxygen-evolving complex of photosystem II the S0 state is oxidized to the S1 state by D+ (signal IIslow). Biochemistry 1987, 26, 2401-2405. [CrossRef]

17. Vass, I.; Styring, S. pH-Dependent charge equilibria between tyrosine-D and the S states in photosystem II. Estimation of relative midpoint redox potentials. Biochemistry 1991, 30, 830-839. [CrossRef]

18. Buser, C.A.; Thompson, L.K.; Diner, B.A.; Brudvig, G.W. Electron-transfer reactions in manganese-depleted photosystem II. Biochemistry 1990, 29, 8977-8985. [CrossRef]

19. Debus, R.J.; Barry, B.A.; Babcock, G.T.; McIntosh, L. Site-directed mutagenesis identifies a tyrosine radical involved in the photosynthetic oxygen-evolving system. Proc. Natl. Acad. Sci. USA 1988, 85, 427-430. [CrossRef] [PubMed] 
20. Vermaas, W.F.; Renger, G.; Dohnt, G. The reduction of the oxygen-evolving system in chloroplasts by thylakoid components. Biochim. Biophys. Acta Bioenerg. 1984, 764, 194-202. [CrossRef]

21. Messinger, J.; Renger, G. Generation, oxidation by the oxidized form of the tyrosine of polypeptide D2, and possible electronic configuration of the redox states S0, S-1, and S-2 of the water oxidase in isolated spinach thylakoids. Biochemistry 1993, 32, 9379-9386. [CrossRef] [PubMed]

22. Ananyev, G.M.; Sakiyan, I.; Diner, B.A.; Dismukes, G.C. A Functional Role for Tyrosine-D in Assembly of the Inorganic Core of the Water Oxidase Complex of Photosystem II and the Kinetics of Water Oxidation. Biochemistry 2002, 41, 974-980. [CrossRef]

23. Boerner, R.; Bixby, K.; Nguyen, A.; Noren, G.; Debus, R.; Barry, B. Removal of stable tyrosine radical D+ affects the structure or redox properties of tyrosine $\mathrm{Z}$ in manganese-depleted photosystem II particles from Synechocystis 6803. J. Biol. Chem. 1993, 268, 1817-1823. [CrossRef]

24. Havelius, K.G.V.; Styring, S. pH Dependent Competition between YZ and YD in Photosystem II Probed by Illumination at 5 K. Biochemistry 2007, 46, 7865-7874. [CrossRef] [PubMed]

25. Deak, Z.; Vass, I.; Strying, S. Redox interaction of Tyrosine-D with the S-states of the water-oxidizing complex in intact and chloride-depleted Photosystem II. Biochim. Biophys. Acta Bioenerg. 1994, 1185, 65-74. [CrossRef]

26. Feyziyev, Y.; Rotterdam, B.J.; Bernát, G.; Styring, S. Electron transfer from cytochrome b559 and tyrosineD to the S2 and S3 states of the water oxidizing complex in photosystem II. Chem. Phys. 2003, 294, 415-431. [CrossRef]

27. Mamedov, F.; Smith, P.J.; Styring, S.; Pace, R.J. Relaxation behaviour of the tyrosine YD radical in photosystem II: Evidence for strong dipolar interaction with paramagnetic centers in the S1 and S2 states. Phys. Chem. Chem. Phys. 2004, 6, 4890-4896. [CrossRef]

28. Rutherford, A.; Boussac, A.; Faller, P. The stable tyrosyl radical in Photosystem II: Why D? Biochim. Biophys. Acta Bioenerg. 2004, 1655, 222-230. [CrossRef]

29. Saito, K.; Ishida, T.; Sugiura, M.; Kawakami, K.; Umena, Y.; Kamiya, N.; Shen, J.R.; Ishikita, H. Distribution of the Cationic State over the Chlorophyll Pair of the Photosystem II Reaction Center. J. Am. Chem. Soc. 2011, 133, 14379-14388. [CrossRef]

30. Diner, B.A.; Bautista, J.A.; Nixon, P.J.; Berthomieu, C.; Hienerwadel, R.; Britt, R.D.; Vermaas, W.F.J.; Chisholm, D.A. Coordination of proton and electron transfer from the redox-active tyrosine, $\mathrm{YZ}$, of Photosystem II and examination of the electrostatic influence of oxidized tyrosine, YD(H+). Phys. Chem. Chem. Phys. 2004, 6, 4844-4850. [CrossRef]

31. Jeans, C.; Schilstra, M.J.; Ray, N.; Husain, S.; Minagawa, J.; Nugent, J.H.A.; Klug, D.R. Replacement of Tyrosine D with Phenylalanine Affects the Normal Proton Transfer Pathways for the Reduction of P680+ in Oxygen-Evolving Photosystem II Particles from Chlamydomonas. Biochemistry 2002, 41, 15754-15761. [CrossRef] [PubMed]

32. Magnuson, A.; Rova, M.; Mamedov, F.; Fredriksson, P.O.; Styring, S. The role of cytochrome b559 and tyrosineD in protection against photoinhibition during in vivo photoactivation of Photosystem II. Biochim. Biophys. Acta Bioenerg. 1999, 1411, $180-191$. [CrossRef]

33. Deligiannakis, Y.; Ivancich, A.; Rutherord, A. 2D-Hyperfine sublevel correlation spectroscopy of tyrosyl radicals. Spectrochim. Acta Part A 2002, 58, 1191-1200. [CrossRef]

34. Mino, H.; Astashkin, A.V.; Kawamori, A. An EPR and pulsed ENDOR study of the structure of tyrosine Z• in Tris-treated photosystem II. Spectrochim. Acta Part A 1997, 53, 1465-1483. [CrossRef]

35. Teutloff, C.; Pudollek, S.; Keßen, S.; Broser, M.; Zouni, A.; Bittl, R. Electronic structure of the tyrosine D radical and the water-splitting complex from pulsed ENDOR spectroscopy on photosystem II single crystals. Phys. Chem. Chem. Phys. 2009, 11, 6715-6726. [CrossRef] [PubMed]

36. Mark, B.; Coates, C.S.; Sugimura, S.; Baldansuren, A.; Ku, J.; Debus, R.J.; Lakshmi, K. Determining the Electronic Structure of Paramagnetic Intermediates in membrane proteins: A high-resolution 2D 1H hyperfine sublevel correlation study of the redox-active tyrosines of photosystem II. Biochim. Biophys. Acta Biomembr. 2020, 1862, 183422. [CrossRef] [PubMed]

37. Warncke, K.; McCracken, J.; Babcock, G.T. Structure of the YD Tyrosine Radical in Photosystem II as Revealed by 2H Electron Spin Echo Envelope Modulation (ESEEM) Spectroscopic Analysis of Hydrogen Hyperfine Interactions. J. Am. Chem. Soc. 1994, 116, 7332-7340. [CrossRef]

38. Chatterjee, R.; Coates, C.S.; Milikisiyants, S.; Lee, C.I.; Wagner, A.; Poluektov, O.G.; Lakshmi, K.V. High-Frequency Electron Nuclear Double-Resonance Spectroscopy Studies of the Mechanism of Proton-Coupled Electron Transfer at the Tyrosine-D Residue of Photosystem II. Biochemistry 2013, 52, 4781-4790. [CrossRef]

39. Sjöholm, J.; Mamedov, F.; Styring, S. Spectroscopic Evidence for a Redox-Controlled Proton Gate at Tyrosine D in Photosystem II. Biochemistry 2014, 53, 5721-5723. [CrossRef]

40. Hasler, L.; Ghanotakis, D.; Fedtke, B.; Spyridaki, A.; Miller, M.; Müller, S.A.; Engel, A.; Tsiotis, G. Structural Analysis of Photosystem II: Comparative Study of Cyanobacterial and Higher Plant Photosystem II Complexes. J. Struct. Biol. 1997, 119, 273-283. [CrossRef] [PubMed]

41. McDermott, A.E.; Yachandra, V.K.; Guiles, R.D.; Cole, J.L.; Dexheimer, S.L.; Britt, R.D.; Sauer, K.; Klein, M.P. Characterization of the manganese oxygen-evolving complex and the iron-quinone acceptor complex in photosystem II from a thermophilic cyanobacterium by electron paramagnetic resonance and x-ray absorption spectroscopy. Biochemistry 1988, 27, $4021-4031$. [CrossRef]

42. Yachandra, V.K.; Sauer, K.; Klein, M.P. Manganese Cluster in Photosynthesis: Where Plants Oxidize Water to Dioxygen. Chem. Rev. 1996, 96, 2927-2950. [CrossRef] 
43. Boussac, A.; Kuhl, H.; Un, S.; Rögner, M.; Rutherford, A.W. Effect of Near-Infrared Light on the S2-State of the Manganese Complex of Photosystem II from Synechococcus elongatus. Biochemistry 1998, 37, 8995-9000. [CrossRef]

44. Zahariou, G.; Ioannidis, N.; Sanakis, Y.; Pantazis, D.A. Arrested Substrate Binding Resolves Catalytic Intermediates in HigherPlant Water Oxidation. Angew. Chem. Int. Ed. 2021, 60, 3156-3162. [CrossRef] [PubMed]

45. Berthold, D.A.; Babcock, G.T.; Yocum, C.F. A highly resolved, oxygen-evolving photosystem II preparation from spinach thylakoid membranes: EPR and electron-transport properties. FEBS Lett. 1981, 134, 231-234. [CrossRef]

46. Ford, R.; Evans, M. Isolation of a photosystem 2 preparation from higher plants with highly enriched oxygen evolution activity. FEBS Lett. 1983, 160, 159-164. [CrossRef]

47. Stoll, S.; Schweiger, A. EasySpin, a comprehensive software package for spectral simulation and analysis in EPR. J. Magn. Reson. 2006, 178, 42-55. [CrossRef] [PubMed]

48. Dikanov, S.A.; Tyryshkin, A.M.; Bowman, M.K. Intensity of Cross-Peaks in Hyscore Spectra of S =1/2, I = 1/2 Spin Systems. J. Magn. Reson. 2000, 144, 228-242. [CrossRef]

49. Dikanov, S.; Bowman, M. Cross-Peak Lineshape of Two-Dimensional ESEEM Spectra in Disordered S = 1/2, I = 1/2 Spin Systems. J. Magn. Reson. Ser. A 1995, 116, 125-128. [CrossRef]

50. Schweiger, A.; Jeschke, G. Principles of Pulse Electron Paramagnetic Resonance; Oxford University Press: New York, NY, USA, 2001.

51. Stoll, S.; Calle, C.; Mitrikas, G.; Schweiger, A. Peak suppression in ESEEM spectra of multinuclear spin systems. J. Magn. Reson. 2005, 177, 93-101. [CrossRef] [PubMed]

52. O' Malley, P.J.; MacFarlane, A.J.; Rigby, S.E.; Nugent, J.H. The geometry and spin density distribution of the tyrosyl radical: A molecular orbital study. Biochim. Biophys. Acta Bioenerg. 1995, 1232, 175-179. [CrossRef]

53. McConnell, H.M.; Chesnut, D.B. Negative Spin Densities in Aromatic Radicals. J. Chem. Phys. 1957, 27, 984-985. [CrossRef]

54. McConnell, H.M. Spin Density Matrices for Paramagnetic Molecules. J. Chem. Phys. 1958, 28, 1188-1192. [CrossRef]

55. Heller, C.; McConnell, H.M. Radiation Damage in Organic Crystals. II. Electron Spin Resonance of $(\mathrm{CO} 2 \mathrm{H}) \mathrm{CH} 2 \mathrm{CH}(\mathrm{CO} 2 \mathrm{H})$ in $\beta$-Succinic Acid. J. Chem. Phys. 1960, 32, 1535-1539. [CrossRef]

56. Fessenden, R.W.; Schuler, R.H. Electron Spin Resonance Studies of Transient Alkyl Radicals. J. Chem. Phys. 1963, 39, $2147-2195$. [CrossRef]

57. Nakazawa, S.; Ishii, A.; Minagawa, J.; Ono, T. Application of 2D-HYSCORE spectroscopy to tyrosine radicals $\mathrm{Y}_{D}$ and $\mathrm{Y}_{Z}$ in photosystem II for evaluation of spin density distributions. Chem. Phys. Lett. 2005, 405, 318-322. [CrossRef]

58. Force, D.A.; Randall, D.W.; Britt, R.D.; Tang, X.S.; Diner, B.A. 2H ESE-ENDOR study of hydrogen bonding to the tyrosine radicals YD.bul. and YZ.bul. of photosystem II. J. Am. Chem. Soc. 1995, 117, 12643-12644. [CrossRef]

59. Keßen, S.; Teutloff, C.; Kern, J.; Zouni, A.; Bittl, R. High-Field 2H-Mims-ENDOR Spectroscopy on PSII Single Crystals: Hydrogen Bonding of YD. ChemPhysChem 2010, 11, 1275-1282. [CrossRef]

60. Gilchrist, M.L.; Ball, J.A.; Randall, D.W.; Britt, R.D. Proximity of the manganese cluster of photosystem II to the redox-active tyrosine YZ. Proc. Natl. Acad. Sci. USA 1995, 92, 9545-9549. [CrossRef]

61. Farrar, C.T.; Gerfen, G.J.; Griffin, R.G.; Force, D.A.; Britt, R.D. Electronic Structure of the YD Tyrosyl Radical in Photosystem II: A High-Frequency Electron Paramagnetic Resonance Spectroscopic and Density Functional Theoretical Study. J. Phys. Chem. B 1997, 101, 6634-6641. [CrossRef]

62. Hofbauer, W.; Zouni, A.; Bittl, R.; Kern, J.; Orth, P.; Lendzian, F.; Fromme, P.; Witt, H.T.; Lubitz, W. Photosystem II single crystals studied by EPR spectroscopy at 94 GHz: The tyrosine radical Y. Proc. Natl. Acad. Sci. USA 2001, 98, 6623-6628. [CrossRef]

63. Fotinou, C.; Kokkinidis, M.; Fritzsch, G.; Haase, W.; Michel, H.; Ghanotakis, D.F. Characterization of a Photosystem II core and its three-dimensional crystals. Photosynth. Res. 1993, 37, 41-48. [CrossRef] [PubMed] 